\title{
Study on the simulation method of architectural design based on 3D virtual modelling
}

WANG Ying-quan

\author{
Sichuan College of Architectural Technology, Sichuan, Deyang 618000,China
}

Keywords: 3D virtual modelling; architecture; design

\begin{abstract}
Architecture design with the use of three-dimensional virtual modelling technology has become the main trend of the architecture field. To this end, a 3D virtual modelling architecture design method based on the model of multilevel texture mapping is proposed. Combining the relevant principles of multilevel analysis and texture mapping relationship model to obtain multilevel texture mapping model, building feature points are input into this model, in order to obtain the feature mapping relationship. According to the characteristics mapping relationship, the architecture design 3D virtual model is established. The experimental results show that, by using the improved algorithm for 3D virtual modelling to meet the actual demand of architecture design.
\end{abstract}

\section{Introduction}

With the 3D virtual technology can obtain the building location distribution in space, so as to realize the architecture design based on 3D virtual modelling [1-2]. Therefore, the method of architectural design based on 3D virtual technology [3], is the core problem to research in the construction field. At present, the mainstream 3D virtual modelling architecture design method mainly includes the method based on features points overlapping algorithm [4], 3D stereo vision technology [5] and image key features algorithm [6]. Among them, the most commonly used is based on the feature points overlap algorithm [7].

The 3D virtual modelling architecture design established with the traditional algorithm, assuming there are no obvious key features in the building image [8], matching errors are caused, resulting in characteristics migration of architecture 3D simulation model, model accuracy is reduced, which reduced the reality of architecture design [9].

In order to avoid the disadvantages of the traditional algorithm, a 3D virtual modelling architecture design method based on the model of multilevel texture mapping is proposed. Combining the relevant principles of multilevel analysis and texture mapping relationship model to obtain multilevel texture mapping model, building feature points are input into this model, in order to obtain the feature mapping relationship. According to the characteristics mapping relationship, the architecture design 3D virtual model is established. The experimental results show that, by using the improved algorithm for 3D virtual modelling to meet the actual demand of architecture design.

\section{The process characteristics of 3D virtual modelling in architecture design}

Analyzing 3D virtual modelling process framework, although every part of the building has different characteristics, there are still common in general, that is, the process structure, technology structure and knowledge structure in 3D virtual modelling process of the architectural design are showing the complexity.

Complexity of building structure design.The 3D virtual modelling process of the architectural design of general complexity is relatively simple in structure, and easy to operate, 3D modelling of more complex architectural design structure will have a corresponding increase in the degree of difficulty, and in the modelling process, often encounter the situation, like additional design or modify the design are needed. In the three-dimensional virtual modelling process of architecture design, modify the program and modify the design are not uncommon cases in the process of 3D virtual modelling of architectural design, which makes the structure of the whole design process more complicated. 
Dynamic and complexity of the technical structure.Three-dimensional virtual model of architectural design is a logical body, do not have specific physical properties, it has the abstract, and must be established on the top of the design and related modelling software. First, there must be good art design. Secondly, a large number of advanced design techniques have to be adopted. Finally, the appropriate software must be used to solve the problem appeared in the design process. Currently, the architectural design three-dimensional virtual modelling software used in industry is various, select the right and program suitable software platform can reduce complexity for project design, improve design efficiency and improve the quality of design. In three-dimensional virtual modelling process of architectural design, many technical encountered bottlenecks in achieving process, which have to be fixed by creativity. Generally speaking, the more innovative modelling performance, the greater the need to resort to technological innovation. In conclusion, the threedimensional virtual modelling technology of the architectural design is evolving, from single to multiple, from lower to higher, from simple to complex, this technology has a clear dynamic and complex nature.

\section{The principles of architectural design method based on 3D virtual modelling}

The establishment of a multi-level texture mapping model.In the process of establishing a multilevel texture mapping model, integration of the related principle of the multi-level analysis and texture mapping model is necessary to reduce the complexity of building structure. By using the building image texture, can effectively improve fineness and authenticity of 3D virtual model of architectural design.

When establishing a multi-level texture mapping model, the main steps are as follows:

(1) establish a single texture mapped model: under the situation of reducing building model complexity in maximum, the geometric structure of building is obtained according to texture image of buildings, multi-level color characteristics of the building will be described in the corresponding geometric structure, so as to improve the authenticity of the 3D virtual model of architectural design.

(2) establish penetrating texture mapping model: the image fusion technology is applied to the process of establishing the texture mapping model, combining the color and texture of different levels, to make the obtained building in translucent state, and fuse with architectural surroundings.

There are many methods of establishing multi-level texture mapping model, the main methods include, spherical feature point mapping method, gray mapping method and color mapping method. In this paper, spherical feature point mapping method is selected for establishment of multi-level texture mapping model. During the process of modelling, the texture of the surfaces of buildings have to be mapped on the spherical surface where 3D models of architectural design located, so as to obtain the texture characteristics of 3D model.

According to the method described above, a multi-level texture mapping model of architectural design can be built, so as to provide the basis for 3D virtual model of architectural design.

Implementation of the 3D virtual modelling of the architecture design. The building characteristic value can be described with $c_{q}$, the spatial position of the corresponding feature point can be described with $y_{q}$, the difference between the different spatial position characteristics point can be described with $\varphi_{q}$. The following formula can be used to calculate the difference of twodimensional image neighborhood characteristics point in the building design:

$$
\bar{z}=\overline{z_{0}}-\left(\frac{m}{m}\right) C \sin (\bar{m})
$$

$y_{q}^{2}=i m_{q}^{2} j$ is substituted into the plane image neighborhood feature point difference calculation formula of architecture design to get the following formula:

$$
\mu(z, a, v)=\sum_{q=1}^{\infty} c_{q} \cos \left[y_{q} v-\sqrt{\frac{y_{q}^{2}}{i j}}\left(z \cos \rho_{q}\right)+\varphi_{q}\right]
$$

In the above formula, $j$ can be used to describe the Euclidean distance between different feature points of the architecture design, $\rho_{q}$ can be used to describe the area of architecture design. 
According to Fourier transform related theory, the spatial location relationship between the feature point in architecture design can be calculated as follows:

$$
\left\{\begin{array}{c}
\frac{\partial w(z, v)}{\partial v}+w(z, v) \cdot \nabla w(z, v)+\nabla r(z, v) / \varsigma= \\
\nabla w(z, v)=0 \\
x \nabla^{2} w(z, v)+H
\end{array}\right.
$$

In the above formula, $w(z, v)$ can be used to describe the gray changes rate of 3D virtual model of architecture design, $r(z, v)$ can be used to describe the feature point space transform function, $\varsigma$ is the coordinate of the feature points.

The spatial position relation of the feature points of 3D virtual model can be calculated by using the following formula:

$$
\begin{aligned}
& \bar{z}=\overline{z_{0}}-\left(\frac{\bar{m}}{m}\right) C \sin (\bar{m} \cdot \bar{z}-y v) \\
& a=C \cos \left(\bar{m} \cdot \overline{z_{0}}-y v\right)
\end{aligned}
$$

According to the method described above, a 3D virtual model designed by architecture can be established so as to realize the architecture design.

\section{Analysis of simulation results}

In order to verify the effectiveness of improved algorithms, there is the need for an experiment. In the experiment process, a 3D virtual modelling of architecture design is established with different algorithms.

With different algorithm to establish the three-dimensional virtual model of architecture design, modelling consumed time can be described with the figure below:

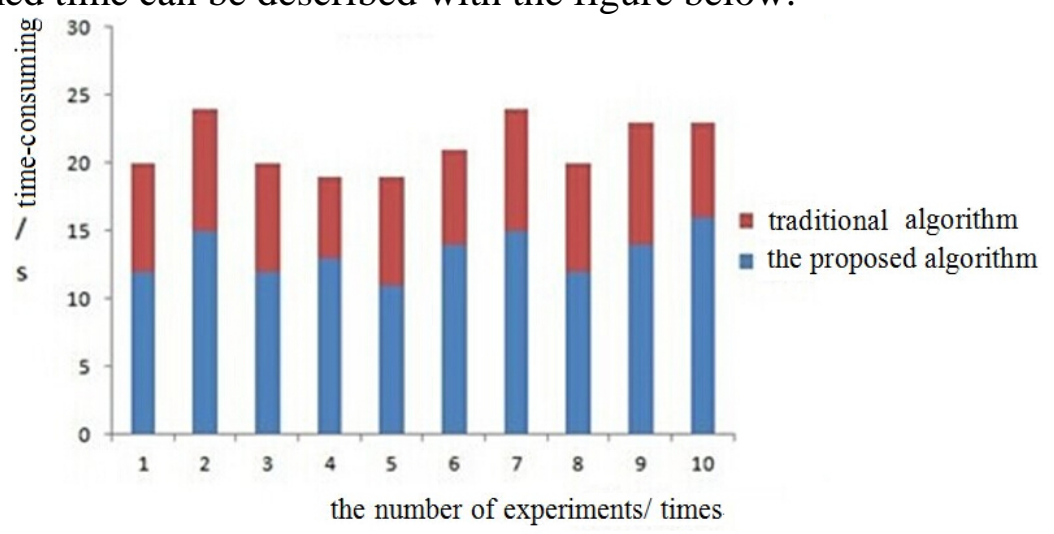

Figure 1 comparison of modelling time-consuming using different algorithms

The above experimental data is analyzed to obtain the results in the following table:

Table 1 data of time-consuming using different algorithms

\begin{tabular}{lll}
\hline The number of experiments & Traditional algorithm $(\mathrm{s})$ & Improved algorithm (s) \\
\hline 1 & 12 & 8 \\
2 & 15 & 9 \\
3 & 12 & 8 \\
4 & 13 & 6 \\
5 & 11 & 8 \\
6 & 14 & 7 \\
7 & 15 & 9 \\
8 & 12 & 8 \\
9 & 14 & 9 \\
10 & 16 & 7 \\
\hline
\end{tabular}


According to the experimental results above we can know that, with the proposed algorithm to process 3D virtual modelling of architecture design, time cost is shorter than the traditional algorithm.

During the process of 3D virtual modelling for architecture design with different algorithms, the reality of the model can be described with the following figure.

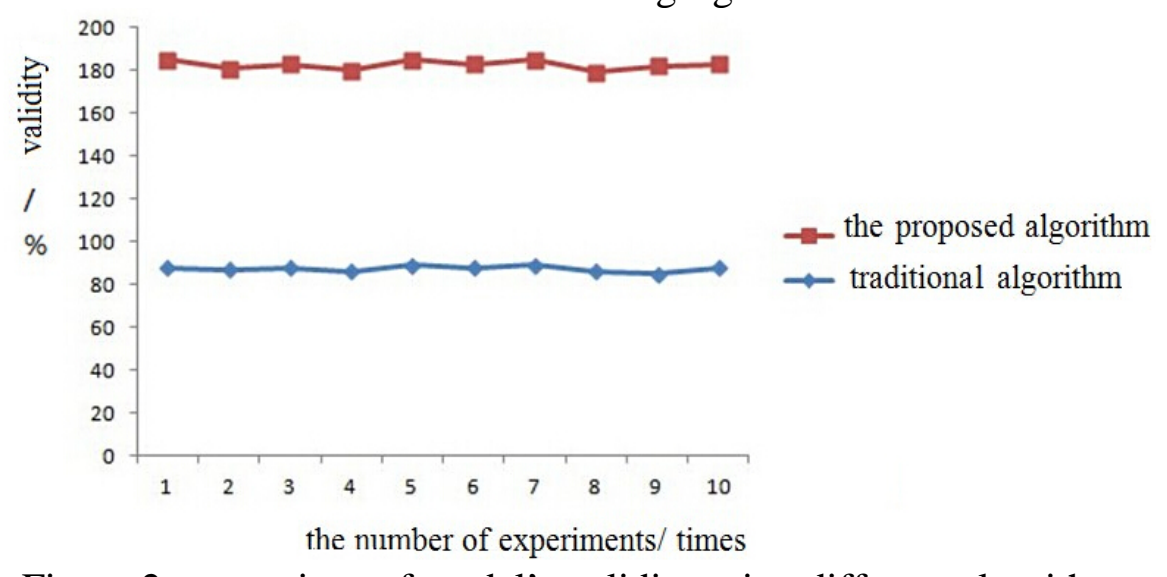

Figure 2 comparison of model's validity using different algorithms

The data in the above experiment process is analyzed, the following results can be obtained:

Table 2 validity results of modelling using different algorithms

\begin{tabular}{lll}
\hline The number of experiments & Traditional algorithm (\%) & Improved algorithm (\%) \\
\hline 1 & 88 & 97 \\
2 & 87 & 94 \\
3 & 88 & 95 \\
4 & 86 & 94 \\
5 & 89 & 96 \\
6 & 88 & 95 \\
7 & 89 & 96 \\
8 & 86 & 93 \\
9 & 85 & 97 \\
10 & 88 & 95 \\
\hline
\end{tabular}

On the basis of the above experiments, it can be learnt that with the proposed algorithm for 3D virtual modelling of buildings, the reality of model is much higher than that of the traditional algorithm, fully demonstrate the advantages of the proposed algorithm.

In the process of experiment, using different algorithms for $3 \mathrm{~d}$ virtual modelling of architectural design, design of customer satisfaction can be described in the table below:

Table 3 different algorithm modelling design of customer satisfaction data tables

\begin{tabular}{lll}
\hline The number of & $\begin{array}{l}\text { Traditional algorithm of customer } \\
\text { satisfaction (\%) }\end{array}$ & $\begin{array}{l}\text { Customer satisfaction improved } \\
\text { algorithm (\%) }\end{array}$ \\
\hline 1 & 96 & 99 \\
2 & 95 & 99 \\
3 & 96 & 98 \\
4 & 94 & 99 \\
5 & 96 & 99 \\
6 & 94 & 98 \\
7 & 95 & 99 \\
8 & 95 & 99 \\
9 & 97 & 97 \\
10 & 95 & 98 \\
\hline
\end{tabular}




\section{Conclusions}

Aiming at the requirements for virtual 3D modelling technology in the architecture design process, a 3D virtual modelling architecture design method based on the model of multilevel texture mapping is proposed. Combining the relevant principles of multilevel analysis and texture mapping relationship model to obtain multilevel texture mapping model, building feature points are input into this model, in order to obtain the feature mapping relationship. According to the characteristics mapping relationship, the architecture design 3D virtual model is established. The experimental results show that, by using the improved algorithm for 3D virtual modelling to meet the actual demand of architecture design.

\section{References}

[1] AN XW, ZHANG XB, YIN HCH. The research of Three-Dimensional medical image reconstruction [J]. Journal of Electron Devices, 2001, 3:207-212.

[2] Barbara Zitová, Jan Flusser. Image registration methods: a survey [J]. Image and Vision Computing: 2003, 21(11): 977-1000.

[3] Wei Chuliang, Luo Peiyu, Hong Xiaobin, Qiu Chaole, Cai Zemin. Research on the new method of three dimensional images reconstruction based on single pictures model [J]. China measurement and test, 2014, 40 (2): 23-26.

[4] J. -S. Chen D. P. Chu.Fast calculation of wave front amplitude propagation: a tool to analyze the 3D image on a hologram （Invited Paper） [J].Chinese Optics Letters, 2014(6):95-98.

[5] Li Zhaohui, Wei Jun, Lv Zhongwen, Tian Yu, Du Chao. Application of 3D anatomic images, video clips and 3D image of clinical imaging in Neurosurgery teaching [J]. Chinese Journal of laboratory diagnosis, 2013, 17 (9): 1734-1735.

[6] Timothy S. Newman, Hong Yi. A survey of the marching cubes algorithm [J].Computers \& Graphics, 2006, 30(5): 854-879.

[7] Schroeder Wj, Avila Ls, Hoffman W. Visualizing with VTK: A tutorial. IEEE Trans [J]. On Computer Graphics and Applications, 2000, 20(5):20-27.

[8] Zhao Feng, Liu Zhenxia, Gao Bo. To establish the three-dimensional finite element model of mandible under Dicom standard and Mimics software [J]. Southwest Military Medicine, 2005, 15 (5): 479-481.

[9] Liu Jun, Wu Helei. The construction of finite element model of real head based on MRI data [J]. Journal of Nanchang Aviation University, 2012, 26 (3): 6-10.

[10] Light, Yao Lixiu, Chen Lu etc. Based on the general model of single face image $3 \mathrm{~d}$ reconstruction [J]. Journal of Shanghai jiaotong university, 2009, 43 (3) : 432-436.

[11] Zhen-xing gao, hong-bin gu. Used for real-time flight simulation under the micro strike critical flow modelling study [J]. Journal of system simulation, 2008, 20 (23) : 6524-6529.

[12] Linda, chang, gen-cai zhu. Meteorological echo characteristics of the stochastic process simulation [J]. Computer simulation, 2007, 24 (12) : 88-93.

[13] Zheng pay league. 3 ds Max modelling and optimization of research [J]. Journal of popular science and technology, 2010, 2:43-44.

[14] Wang Wuli, yan-jiang wang, Yang hua, and so on. $3 \mathrm{~d}$ visualization technology in the application of drilling simulation [J]. Journal of engineering graphics, 2006; 28 (6) : 47-5.

[15] Feng Yufen. Virtual community based on Virtools roaming system [J]. The design and implementation of computer simulation, 2009; 26 (6) : 285-287. 